\title{
ON FINITE INVARIANT MEASURES FOR SETS OF MARKOV OPERATORS
}

\author{
S. HOROWITZ ${ }^{1}$
}

\begin{abstract}
A. Brunel [1] proved that a Markovian operator $P$ has an invariant measure if and only if each convex combination of iterates $\sum_{n=0}^{\infty} \alpha_{n} P^{n}$ is conservative. In the present paper this result is generalized for any commutative semigroup of Markovian operators: Let $\Pi$ be a semigroup; there exists a common invariant measure for $\Pi$ if and only if each convex combination $\sum_{n=1}^{\infty} \alpha_{n} P_{n}$, where $\left\{P_{n}\right\} \subset \Pi$, is conservative.
\end{abstract}

1. Definitions and notations. Let $(X, \Sigma, m)$ be a finite measure space. A Markov operator $P$ is a positive contraction on $L_{1}(X, \Sigma, m)$ i.e., (i) $\|P\| \leqq 1$, (ii) $u \geqq 0 \Rightarrow u P \geqq 0$. We shall use the notations of [3], so the operator adjoint to $P$ which is defined in $L_{\infty}(m)$ will also be denoted by $P$ to the left side of the variable. Thus $\langle u P, f\rangle=\langle u, P f\rangle, u \in L_{1}(m), f \in L_{\infty}(m)$. We denote $\Sigma_{i}(P)=\left\{A \in \Sigma \mid P 1_{A}=1_{A}\right.$ a.e. $\}$. If $P$ is conservative, then $\Sigma_{i}(P)$ is a field.

Let us consider the commutative semigroup $\Pi$ of conservative Markov operators. The invariant sets of $\Pi$ is the collection $\Sigma_{i}=\bigcap_{P \in \Pi} \Sigma_{i}(P)$.

Let $\Pi$ be the convex hull of $\Pi$ : (i) $\Pi \subset \Pi$, (ii) $\left\{P_{n}\right\} \subset \Pi$ and $P_{n} \rightarrow P$ in operator norm then $P \in \Pi$. (iii) $P_{1}, P_{2} \in \Pi, 0<a, \beta<1$ and $\alpha+\beta=1$ then $\alpha P_{1}+\beta P_{2} \in \Pi$. (iv) $\Pi$ is minimal under those conditions.

A measure $\mu$ is said to be invariant for $\Pi$ if $\mu P=\mu$ for every $P \in \Pi$. Clearly, $\Pi$ is a semigroup and if $\mu$ is a finite invariant measure for $\Pi$ it is invariant for $\Pi$.

In this paper we prove that if there is no finite invariant measure for $\Pi$ then there exists an operator $Q \in \Pi$ such that the dissipative part of $Q, D=X$. (For definitions see [3].)

REMARK. In [1] it is proved for $\Pi=\left\{P^{n}\right\}$, where $P$ is a given Markov operator, that if it has no finite invariant measures, then there exists $Q \in \Pi$, such that the dissipative part of $Q, D \neq \varnothing$.

Received by the editors September 16, 1971.

AMS 1970 subject classifications. Primary 47A35; Secondary 28A65.

Key words and phrases. Commutative semigroups, Markov operators, invariant measures, conservative processes, dissipative processes, convex combinations, positive combination on $L_{\infty}$.

${ }^{1}$ Partially supported by National Science Foundation Grant GU 3171 and a State University of New York Faculty Fellowship.

(c) American Mathematical Society 1972 


\section{Conservative operators and invariant measures.}

LEMMA 2.1. If there are no finite invariant measures for $\Pi$, then there exists $\left\{P_{n}\right\} \subset \Pi$ and $0 \leqq f \in L_{\infty}(m)$ such that $\sum_{n=1}^{\infty} P_{n} f \in L_{\infty}(m)$.

Proof. In [4] it is proved that if there exists no finite invariant measure then there exists $0 \leqq g \in L_{\infty}(m)$ such that $\inf \left\{\int P g d m \mid P \in \Pi\right\}=0$. Hence by slight modifications of the proof of Lemma C, Chapter IV of [3], or of the more elegant proof of this lemma which appears in [2], Lemma 2.1 can be proved.

Let us define the space:

$$
L=\operatorname{spn}\left\{(I-P) L_{\infty}(m) \mid P \in \Pi\right\} .
$$

The orthogonal complement of $L$ is

$$
L^{\perp}=\left\{v \in L_{\infty}^{*}(m) \mid v P=v \forall P \in \Pi\right\} .
$$

$L_{\infty}^{*}(m)$ is the space of the charges (finitely additive measures). If $P 1=1$, as. it is in the conservative case, then $\nu P=v$ implies $\nu^{+} P=\nu^{+}$. Define:

$$
M=\left\{v \in L_{\infty}^{*}(m) \mid v \geqq 0,\|v\|=1, \nu P=v \forall P \in \Pi\right\} .
$$

It is easy to show that:

$$
f \in L \Leftrightarrow\langle v, f\rangle=0, \quad v \in M .
$$

LEMMA 2.2. If there exists no finite invariant measures for $\Pi$, then there exists $0 \neq f \geqq 0$ such that $f \in L$.

Proof. Let $f$ be the function of Lemma 2.1. It is clear that $\langle v, f\rangle=0$ or each $\nu \in M$ and by (2.4), $f \in L$. .

LEMMA 2.3. $X$ may be decomposed uniquely into the disjoint union $X=X_{0} \cup X_{1}$ where (i) $X_{0}, X_{1} \in \Sigma_{i}$. (ii) There exists a finite invariant measure for $\Pi$ equivalent to $m I_{X_{1}}$. (iii) There exists $\left\{A_{n}\right\} \subset \Sigma$ with $A_{n} \nearrow X_{0}$, and $1_{A_{n}} \in L, \forall{ }_{n}$.

Proof. Let $\mu$ be any finite invariant measure for $\Pi$, let $B=\operatorname{supp} \mu$; it is easy to see that $B \in \Sigma_{i}$. Let $\alpha=\sup \{m(B) \mid B=\operatorname{supp} \mu, \mu$ a finite invariant measure for $\Pi\}$. Hence there exists a sequence of finite invariant measures $\left\{\mu_{n}\right\}$, such that $m\left(B_{n}\right) \nearrow \alpha_{\infty}$, where $B_{n}=\sup \mu_{n}$. Define $X_{1}=\bigcup_{n=1}^{\infty} B_{n}$ and $\lambda=\sum_{n=1}^{\infty}\left(1 / 2^{n}\right) \mu_{n}$ and then $\lambda$ is a finite invariant measure with supp $\lambda=$ $x_{1}, m\left(X_{1}\right)=\alpha$, and $X_{1} \in \Sigma_{i}$ (or $P 1_{X_{1}}=1_{X_{1}}, \forall P \in \Pi$ ). Define $X_{0}=X-X_{1}$, assume that there exists a finite invariant measure for $\Pi, \lambda^{\prime}$ supported on $X_{0}$. Let supp $\lambda^{\prime}=B^{\prime} \subset X_{0}$, then $\lambda+\lambda^{\prime}$ is a finite invariant measure for $\Pi$, $\operatorname{supp}\left(\lambda+\lambda^{\prime}\right)=X_{1} \cup B^{\prime}$ and $m\left(X_{1} \cup B^{\prime}\right)>\alpha$, a contradiction. 
Since $X_{0} \in \Sigma_{i}$ (and $P 1_{X_{0}}=1_{X_{0}}$ ) we can restrict the Markov operators of $\Pi$ to $\left(X_{0}, \Sigma_{X_{0}}, m I_{X_{0}}\right)$, and apply Lemma 2.2. Formula (2.4) implies that if $0 \leqq g \leqq f$ and $f \in L$ and $g \in L$, and if $0 \leqq f, g \in L$, then $\max (f, g) \in L$. Let $\mathfrak{U}=\left\{A \mid 1_{A} \in L\right\}$; clearly if $0 \leqq f \in L$ and $A=\{f \geqq \varepsilon>0\}$ then $A \in \mathfrak{A}$, if $A \in \mathfrak{A}$ and $B \subset A$ then $B \in \mathfrak{A}$, and if $A, B \in \mathfrak{A}$ then $A \cup B \in \mathfrak{A}$. Let $\beta=$ $\sup \{m(A) \mid A \in \mathfrak{U}\}$. There exists a sequence $\left\{A_{n}\right\}$ such that $m\left(A_{n}\right) \nearrow \beta$. It can be supposed that $A_{n} \subset A_{n+1}, \forall_{n}$ (if not, replace $A_{n}$ by $A_{n}^{\prime}=\bigcup_{k=1}^{n} A_{n}$, and then $A_{n}^{\prime} \in \mathfrak{A}$ and $\left.m\left(A_{n}^{\prime}\right) \nearrow \beta\right)$. Let $A_{n} \nearrow X_{0}^{\prime}$. If $X_{0}^{\prime} \notin \Sigma_{i}$ then there exists $P \in \Pi$ such that $\left\{P 1_{X_{0}{ }^{\prime}}>0\right\} \notin X_{0}^{\prime}$, and hence $\exists_{n}, \varepsilon>0$ such that $\left\{P 1_{A_{n}}>\varepsilon\right\} \notin$ $X_{0}^{\prime}$. Let $E=\left\{P 1_{A_{n}}>\varepsilon\right\}, 1_{E} \in L$ by (2.4), because for each $v \in M$ we have:

$$
\left\langle v, 1_{B}\right\rangle \leqq(1 / \varepsilon) \cdot\left\langle v, P 1_{A_{n}}\right\rangle=(1 / \varepsilon) \cdot\left\langle v, 1_{A_{n}}\right\rangle=0 .
$$

Denote $A_{n}^{\prime}=A_{n} \cup E, A_{n}^{\prime} \in \mathfrak{A}$ and $m\left(A_{n}^{\prime}\right) \nearrow m\left(X_{0}^{\prime} \cup E\right)>\beta$, a contradiction, hence $X_{0}^{\prime} \in \Sigma_{i}$.

If $X_{0}^{\prime} \neq X_{0}$, then we can restrict the Markov operators of $\Pi$ to $\left(X_{0}-X_{0}^{\prime}\right.$, $\left.\Sigma_{X_{0}-X_{0}{ }^{\prime}}, m I_{X_{0}-X_{0}{ }^{\prime}}\right)$, and by Lemma 2.2 there exists $\mathfrak{A} \ni E \subset X_{0}-X_{0}^{\prime}$, denote $A_{n}^{\prime}=A_{n} \cup E, A_{n}^{\prime} \in \mathfrak{U}$ and $m\left(A_{n}^{\prime}\right) \nearrow m\left(X_{0}^{\prime} \cup E\right)>\beta$, a contradiction. So, $X_{0}^{\prime}=X_{0}$ and Lemma 2.3 is proved.

LEMMA 2.4. Let $\left\{A_{n}\right\}$ be the sequence of Lemma 2.3, part (iii), then for each $n$ and for each $\varepsilon>0$, there exists an operator $Q \in \Pi$ such that $\left\|Q 1_{A_{n}}\right\|_{\infty}<$ $\varepsilon$.

Proof. $1_{A_{n}} \in L$, hence there exist $f_{1}, f_{2}, \cdots, f_{j} \in L_{\infty}$ and $P_{1}, P_{2}, \cdots$, $P_{j} \in \Pi$ such that

$$
\left\|\left(f_{1}-P_{1} f_{1}\right)+\left(f_{2}-P_{2} f_{2}\right)+\cdots+\left(f_{j}-P_{j} f_{j}\right)-1_{A_{n}}\right\|<\varepsilon / 2 .
$$

Hence:

$$
\begin{aligned}
& \left\|\frac{1}{N^{j}} \sum_{i_{1}=1}^{N} \sum_{i_{2}=1}^{N} \cdots \sum_{i_{j}=1}^{N} P_{1}^{i_{1}} P_{2}^{i_{2}} \cdots P_{j}^{i_{1}} 1_{A_{n}}\right\|_{\infty} \\
& \leqq
\end{aligned}
$$


But

$$
\begin{aligned}
& \left\|\frac{1}{N^{j}} \sum_{i_{1}=1}^{N} \sum_{i_{2}=1}^{N} \cdots \sum_{i_{k}=1}^{N} \cdots \sum_{i_{j}=1}^{N} P_{1}^{i_{1}} P_{2}^{i_{2}} \cdots P_{k}^{i_{k}} \cdots P_{j}^{i_{j}}\left(f_{k}-P_{k} f_{k}\right)\right\|_{\infty} \\
& =\| \frac{1}{N^{j-1}} \sum_{i_{1}=1}^{N} \sum_{i_{2}=1}^{N} \cdots \sum_{i_{k=1}=1}^{N} \sum_{i_{k+1}=1}^{N} \cdots \sum_{i_{j}=1}^{N} P_{1}^{i_{1}} P_{2}^{i_{2}} \cdots P_{k-1}^{i_{k}-1} P_{k+1}^{i_{k+1}} \cdots P_{j}^{i_{k}} \\
& \times\left[\frac{1}{N} \sum_{i k=1}^{N} P_{k}^{i_{k}}\left(f_{k}-P_{k} f_{k}\right)\right] \|_{\infty} \\
& \leqq 2\left\|f_{k}\right\|_{\infty} / N,
\end{aligned}
$$

if $N$ is sufficiently large then $2\left\|f_{k}\right\|_{\infty} / N \leqq \varepsilon / 2 j$ for $1 \leqq k \leqq j$. Let

and then $\left\|Q 1_{A_{n}}\right\|<\varepsilon$.

$$
Q=\frac{1}{N^{j}} \sum_{i_{1}=1}^{N} \sum_{i_{2}=1}^{N} \cdots \sum_{i_{j}=1}^{N} P_{1}^{i_{1}} P_{2}^{i_{2}} \cdots P_{j}^{i_{j}}
$$

LEMMA 2.5. Let $\left\{A_{n}\right\}$ be the sequence of Lemma 2.3, part (iii), then there exists an operator $V \in \prod$ such that $\lim _{k \rightarrow \infty}\left\|V^{k} l_{A_{n_{k}}}\right\|_{\infty}=0, \forall n$.

Proof. By Lemma 2.4 it can be shown that there exists a sequence of operators $\left\{Q_{n}\right\} \subset \Pi$ such that $\left\|Q_{n} 1_{A_{n}}\right\| \leqq 1 / n$. Let $\alpha_{n}$ be positive numbers such that $\sum_{n=1}^{\infty} \alpha_{n}=1$. Let $V=\sum_{n=1}^{\infty} \alpha_{n} Q_{n}$, then $V \in \Pi$. Given an integer $N$, denote $\beta=\sum_{n=1}^{N} \alpha_{n}, \quad \gamma=\sum_{n=N+1}^{\infty} \alpha_{n}, \beta+\gamma=1$. Define the operators $R=(1 / \beta) \sum_{n=1}^{N} \alpha_{n} Q_{n}$ and $S=(1 / \gamma) \sum_{n=N+1}^{\infty} \alpha_{n} Q_{n}, R, S \in \Pi, \beta R+\gamma S=V$, and $\left\|S 1_{A_{N}}\right\|_{\infty} \leqq 1 / N$. Hence

$$
\left\|V^{k} 1_{A_{N}}\right\|_{\infty}=\left\|(\beta R+\gamma S)^{k} 1_{A_{N}}\right\| \leqq \beta^{k}\left\|R^{k} 1_{A_{N}}\right\|_{\infty}+\left\|S 1_{A_{N}}\right\|_{\infty} \leqq \beta^{k}+1 / N .
$$

Thus for $k$ sufficiently large we have for each $1 \leqq n \leqq N,\left\|V^{k} 1_{A_{n}}\right\|_{\infty} \leqq 2 / N$ but $N$ is arbitrary, hence $\lim _{k \rightarrow \infty}\left\|V^{k} 1_{A_{n}}\right\|_{\infty}=0, \forall n$.

THEOREM. Let $X=X_{0} \cup X_{1}$ be as in Lemma 2.3. Then there exists an operator $U \in \Pi$ such that $X_{1}$ and $X_{0}$ are the conservative and dissipative parts, respectively, for $U$.

Proof. Let $V$ be as in Lemma 2.5. Define the sequence of integers $\left\{n_{k}\right\}$ inductively:

$$
\begin{aligned}
& n_{1}=1, \quad n_{k+1}=n_{k}+1, \quad\left\|V^{j} 1_{A_{n_{k+1}}}\right\|_{\infty} \leqq \frac{1}{n_{k}+1}, \quad \forall j \geqq k+1, \\
& =n_{k}, \\
& \text { otherwise. }
\end{aligned}
$$

Clearly $n_{k} \nearrow \infty$, and $\lim _{k \rightarrow \infty}\left\|V^{k} 1_{A_{n_{k}}}\right\|=0$, where $\left\{A_{n}\right\}$ is the sequence of Lemma 2.3,part (iii). Let $\left\{c_{k}\right\}$ be the sequence of Lemma 3 of [1] such that $\sum_{k=0}^{\infty} c_{k}\left\|V^{k} 1_{A_{n_{k}}}\right\|_{\infty}<\infty$. It is obvious that $\sum_{k=0}^{\infty} c_{k}\left\|V^{k} 1_{A_{n}}\right\|_{\infty}<\infty$ for each $n$. 
Now let $\left\{a_{k}\right\}$ be the sequence associated with $\left\{c_{k}\right\}$ as in Lemma 2 of [1] and let $U=\sum_{k=0}^{\infty} a_{k} V^{k}$, then $U \in \Pi$ and the proof of Theorem 1 of [1] shows that $\sum_{k=0}^{\infty} U^{k} 1_{A_{n}} \in L_{\infty}$ for each $n$, hence $X_{0}=\bigcup_{n=1}^{\infty} A_{n}$ is contained in the dissipative part with respect to $U$. On the other hand if $\mu$ is a finite invariant measure for $\Pi$, supported on $X_{1}$, then $\mu U=\mu$ and $X_{1}$ is contained in the conservative part with respect to $U$.

\section{REFERENCES}

1. A. Brunel, New conditions for existence of invariant measures in ergodic theory, Proc. Conference Contributions to Ergodic Theory and Probability (Ohio State Univ., Columbus, Ohio, 1970), Springer, Berlin, 1970, pp. 7-17. MR 42 \#3253.

2. - Thesis, University of Paris, Paris.

3. S. R. Foguel, The ergodic theory of Markov processes, Van Nostrand Math. Studies, no. 21, Van Nostrand Reinhold, New York, 1969, MR 41 \#6299.

4. M. Lin, Semi-groups of Markov operators. (to appear).

Department of Mathematics, State University of New York at Albany, Albany, New York 12203

Current address: Department of Mathematics, Tel-Aviv University, Tel-Aviv, Israel 